\title{
Cryptanalysis of an Identification Scheme Based on the Permuted Perceptron Problem
}

\author{
Lars R. Knudsen ${ }^{1}$ and Willi Meier ${ }^{2}$ \\ 1 Department of Informatics, University of Bergen, N-5020 Bergen \\ 2 FH-Aargau, CH-5210 Windisch
}

\begin{abstract}
This paper describes an attack on an identification scheme based on the permuted perceptron problem (PPP) as suggested by Pointcheval. The attack finds the secret key, a vector of $n$ binary elements, in time much faster than estimated by its designer. The basic idea in the attack is to use several applications of a simulated annealing algorithm and combine the outcomes into an improved search. It is left as an open problem to what extent the methods developed in this paper are useful also in other combinatorial problems.
\end{abstract}

Keywords: Cryptanalysis. Identification Scheme. Perceptron Problem. Simulated Annealing.

\section{Introduction}

Since the advent of zero-knowledge proofs in 1985 [3], several interactive identification schemes have been proposed. The first protocols, like the Fiat-Shamir scheme 2], were based on number theoretic problems and used arithmetic operations with large numbers. In 1989, Shamir proposed a protocol of a different nature, based on the hardness of an NP-complete problem, the Permuted Kernel Problem, 8]. The distinctive features of this scheme are its use of small integers and its low requirement in memory and processing power. This makes the protocol more suitable for implementations on small processors like smart cards. In the sequel, two new problems (Syndrome Decoding and Constrained Linear Equations) were proposed by Stern, 9], [10]. More recently, Pointcheval presented identification schemes based on the so called Permuted Perceptron Problem (PPP), [5]. PPP is derived from the apparently simpler but still NPcomplete Perceptron Problem (PP), which in turn is motivated by the well known Perceptron in Neural Computing. The identification schemes based on (PPP) are attractive as the operations needed are only additions and subtractions of integers of less than a byte. Thus the schemes are particularly well suited for implementation on 8-bit processors. In view of implementations with restricted memory and/or processing power, a precise determination of the security of the identification schemes is required.

The security of Shamir's and Stern's combinatorial schemes have repeatedly been the subject of publications (see e.g. [7] and the references quoted there). 
The aim of this paper is to investigate the security of Pointcheval's schemes for the parameter values suggested in [5]. The main conclusion is that the smallest parameter values mentioned in $[5],(m=101, n=117)$, are not secure enough for cryptographic applications. For some technical reasons, all parameters proposed in 5] are odd numbers.

In [5], several attacks against PPP have been tried. The most successful method was a probabilistic search, known as simulated annealing. As any solution for PPP is a solution for PP, simulated annealing is applied for solving PP sufficiently often, until a solution that also satisfies PPP may be found. It is reported in [5], that using this method, no solution for PPP for parameter sizes greater than 71 could be found, even if the search continued for long time. As a consequence of the investigations in [5], the parameters $m=101, n=117$ were suggested as a secure size for problem PPP.

In this paper, improved search algorithms to solve PP and PPP are developed. In both problems, a solution consists of a vector of size $n$ with values +1 and -1 as entries. Our main aim is to adapt simulated annealing in several ways to directly solve PPP. Along this way, experiments revealed some intrinsic structure in the problem which enables to turn simulated annealing into an iterated search procedure until a solution may be found. Our algorithms turn out to be successful for instances of PPP for parameter sizes as large as 101 or larger. In particular, our methods are able to solve instances of the target case $m=101, n=117$. Even if we find a solution only in a fraction of cases for these parameters, our algorithm always identifies a subset of entries of a solution vector which are correct with high probability. Besides test results for solving example instances, our algorithms apply to give bounds for solving average instances: We can generally solve PP and thus also PPP up to 280 times faster than was estimated in 5 .

In view of these results it is advisable for secure applications to choose the higher parameter values proposed in [5]. But then the efficiency of the schemes based on PPP compares less favourably to that of the other combinatorial schemes.

It is conceivable that the iterated search method as developed in this paper is also useful in certain other combinatorial and optimization problems.

\section{The Permuted Perceptron Problem}

We follow the notation in [5] where possible. A vector whose entries have value either +1 or -1 is called $\epsilon$-vector, and similarly for matrices.

If $X$ is a vector of size $m$, let $X_{i}$ denote the $i$ th entry in $X$.

Definition 1. The Perceptron Problem $\mathbf{P P}$

Input : An $m \times n \epsilon$-matrix, $A$.

Problem : Find $\epsilon$-vector $V$ of size $n$, such that

$(A V)_{i} \geq 0$, for all $i=1, \ldots, m$. 
In [5] reference is made to [6], showing that $\mathrm{PP}$ is an NP-complete problem. It is possible to design a zero-knowledge identification protocol with every NPcomplete problem provided one-way hash functions exist. However such a protocol will be efficient only if the underlying problem is hard already for moderately sized input parameters. Therefore in [5] the following variant of PP is proposed:

\section{Definition 2. The Permuted Perceptron Problem PPP}

Input $\quad:$ An $m \times n \epsilon$-matrix, $A$, a multiset $S$ of nonnegative numbers of size $m$

Problem : Find $\epsilon$-vector $V$ of size $n$, such that $\left\{\left\{(A V)_{i} \mid i=\{1, \ldots, m\}\right\}\right\}=S$.

Obviously, a solution for PPP is a solution for PP. In 5] this lead to the conclusion that the Permuted Perceptron Problem is more difficult to solve than the original Perceptron Problem. However, knowledge of the prescribed multiset $S$ may give some hint to a solution of PPP. As will be shown in this paper, this is partially the case.

For cryptographic applications, one needs instances for which a solution is known. To get such instances, a (pseudo-) random $\epsilon$-vector $V$ of size $n$ is chosen which will be a solution of the future instance. Hereafter a random $\epsilon$-matrix $A$ of size $m \times n$ is generated and modified in the following way:

For $i=1, \ldots, m$

- If $(A V)_{i}<0$, the $i^{\text {th }}$ row of $A$ is multiplied by -1 .

- If $(A V)_{i} \geq 0$, the $i^{\text {th }}$ row of $A$ remains unchanged.

Finally the multiset $S=\left\{\left\{(A V)_{i} \mid\{i=1, \ldots, m\}\right\}\right\}$ is computed. Consequently, $(A, S)$ is an instance of the Permuted Perceptron Problem, with $V$ as a solution.

In an identification protocol, each prover uses a public instance $(A, S)$, and a secret key $V$. To convince a verifier of his identity, a prover gives him evidence of a solution $V$ to the instance $(A, S)$ by using a zero-knowledge protocol. The description of several such protocols based on PPP is the main subject of [5] and is not detailed here.

\subsection{Simulated Annealing for PP and PPP}

In [5] several attacks against the two problems PP and PPP have been tried in order to evaluate the security of identification protocols based on PPP. Thereby no structure was found which would enable to solve this problem with obvious methods like Gaussian elimination. The attacks made in [5] are outlined subsequently as far as they are relevant to our investigations:

Let $A=\left(a_{i, j}\right)$ be an $m \times n \epsilon$-matrix and let $s_{j}=\sum_{i=1}^{m} a_{i, j}$ for $j=1, \ldots, n$. In a first attempt to attack PPP, the majority vector $M$ is considered, where $M_{j}=\operatorname{sign} s_{j}, j=1, \ldots, n$. As in applications $m$ and $n$ are odd, $M_{j}$ is well defined. 
Suppose $(A, S)$ is an instance for PPP with solution $V$ as considered in the previous section, where $A$ is an $m \times n$ - matrix with $n \geq m$. Then in ([5], Theorem 4) it is stated that the vectors $M$ and $V$ are correlated:

$$
\#\left\{j \mid M_{j}=V_{j}, j=1, \ldots, n\right\} \approx 0.8 n
$$

This leads to a reduced search for a solution $V$ of complexity of order $\left(\begin{array}{c}n \\ 0.2 n\end{array}\right)$. However this number exceeds the common security margin $2^{64}$ for $n \geq 95$.

Due to the inefficiency of the previous attack, in [5] the method of simulated annealing (SA) is proposed. This optimization method simulates a physical cooling process and has been applied for various combinatorial and engineering problems (see e.g., [1], 4]). The idea is to minimize in a probabilistic way an appropriate energy or cost function on a finite space of input variables which has to be provided with a distance measure. SA can only be expected to be efficient if the energy function is roughly continuous, i.e., if the energy difference for neighbouring inputs is bounded by a small number. In [5] the energy function for solving $\mathrm{PP}$ is chosen to be

$$
E(V)=\frac{1}{2} \sum_{i=1}^{m}\left(\left|(A V)_{i}\right|-(A V)_{i}\right)
$$

Figure 1 shows an example of a simulated annealing algorithm for PP and PPP.

Let $\alpha>1$ and $0<\beta<1$. Let $r n d(0,1)$ be a function which returns a random number between 0 and 1 . Choose a candidate vector $V^{\prime}$ at random.

1. Calculate $E\left(V^{\prime}\right)$

2. If $E\left(V^{\prime}\right)=0$ stop

3. $T=\alpha$

4. while $T>1$ do

(a) repeat $n$ times

i. Set $V^{\prime \prime}=V^{\prime}$ and change the sign of one randomly chosen entry of $V^{\prime \prime}$

ii. if $E\left(V^{\prime \prime}\right)<E\left(V^{\prime}\right)$ then $V^{\prime}=V^{\prime \prime}$ else if $\exp \left(\left(E\left(V^{\prime}\right)-E\left(V^{\prime \prime}\right)\right) / T\right)>\operatorname{rnd}(0,1)$ then $V^{\prime}=V^{\prime \prime}$

iii. if $E\left(V^{\prime}\right)=0$ stop

(b) $T=\beta * T$

Fig. 1. Algorithm for simulated annealing for PP and PPP.

Clearly, $E(V)$ is minimum (i.e. $E(V)=0$ ), if and only if the candidate vector $V$ is a solution for PP. For this energy function, SA is successful for instances of size up to 200. On the other hand it is reported in [5] that this way no solution for PPP could be found for instances of size larger than 71, even if the algorithm has been tried for a few months. Hereby, PPP is attacked via solving PP, in the hope of finding a solution for PPP by performing the above SA algorithm sufficiently often. In order to estimate the complexity of such a procedure, the 
approximate number of solutions for PP and for PPP for average instances has been determined. This suggested that the complexity for solving PPP via PP is maximal if $n \approx m+16$, in a practically relevant range $100<m<200$. As a result, three candidate sizes for instances of PPP are proposed in [5]. The smallest size recommended for the matrix $A$ is $m=101, n=117$. It is concluded that solving instances of this size would need 12650 years, corresponding to a complexity of about $2^{64}$ elementary operations, hence a work load sufficient to guarantee the security of the underlying protocol.

\section{Algorithms for Solving PPP}

In this section it is shown how to improve the simulated annealing search for a solution of PPP. In general, the success of this search method essentially depends on the choice of a suitable energy function which is deeply connected to the underlying problem. In order to find such an energy function for PPP, let an $m \times n \epsilon$-matrix $A$ and $Y=A V$ be given as before. Determine a histogram vector $H$ over the integers such that $H_{i}=\#\left\{Y_{j}=i \mid j=1, \ldots, m\right\}$. With $m, n$ odd, $H_{i}$ is set only for odd values of $i, 1 \leq i \leq n$. In a simulated annealing search let $V^{\prime}$ denote the candidate for the secret key $V$, let $Y^{\prime}=A V^{\prime}$ and let $H_{i}^{\prime}$ denote the histogram vector of $Y^{\prime}$. Then an obvious proposal for an energy function would be the distance (in a suitable sense) between the correct and the candidate histogram vector: $E(V)=\sum_{i=1}^{n}\left(\left|H_{i}-H_{i}^{\prime}\right|\right)$ (where the summation index $i$ is only taken over odd values). For this definition of energy, certainly $E(V)=0$ if and only if $V$ is a solution for PPP. Experiments have shown however, that this function $E$ is of no use for a search. The reason is that $E(V)$ may change too much even if only a single sign in $V$ is changed. Hence this choice of $E(V)$ is not continuous as is necessary for simulated annealing to work. As a consequence, one may try to combine this function with the function which has already shown to be successful for PP. This motivates an energy function of the form

$$
E(V)=g_{1} \sum_{i=1}^{m}\left(\left|\left(A V^{\prime}\right)_{i}\right|-\left(A V^{\prime}\right)_{i}\right)+g_{2} \sum_{i=1}^{n}\left(\left|H_{i}-H_{i}^{\prime}\right|\right)
$$

The first part of this function is a multiple of the sum of all negative entries $Y_{i}^{\prime}$, the second part is a multiple of the distance between the correct and candidate histogram vector. It is clear that a solution for PPP (and PP) has been found when $E(V)=0$. The values of $g_{1}$ and $g_{2}$ provide a weighting of the two sums. It has proved useful to choose $g_{1} \geq 30$ and $g_{2}=1$. This introduces additional energy (or penalty) for candidate vectors resulting in negative entries in $Y^{\prime}$ and such candidate vectors never result in a solution for PPP.

Also, the following equation can be used to increase the probability of success of the PPP-search algorithm. 
Corollary 3. Let $A=\left(a_{i, j}\right)$ be an $m \times n$-matrix. Let $s_{j}=\sum_{i=1}^{m} a_{i, j}$ for $j=1, \ldots, n$. If $A V=Y$ then

$$
\sum_{j=1}^{n} s_{j} \cdot V_{j}=\sum_{i=1}^{m} Y_{i} .
$$

Note that the righthand side of Eq. 4 is equal to the sum of the elements in the multiset $S$.

As mentioned in the previous section, the majority vector is in accordance with $V$, the secret key, for about $80 \%$ of the entries. As before, let $s_{j}$ be the vector computed as the sum of every column of $A$. By inspection of the majority vector and the solution vector it follows that this fraction increases for larger values of $\operatorname{abs}\left(s_{j}\right)$. As an example, for instances of PPP where $m=n=73$, experiments show that

$$
\operatorname{Prob}\left(M_{j}=V_{j} \mid \operatorname{abs}\left(s_{j}\right) \geq 11\right) \approx 0.94
$$

whereas for $m=n=101$ this probability is still 0.92 . Moreover for $m=n=73$ and $m=n=101$ tests show that on the average $\operatorname{abs}\left(s_{j}\right) \geq 11$ for 28 respectively 46 entries, or $38 \%$ respectively $46 \%$ of the entries. Similarly for $m=101, n=117$ experiments show that

$$
\operatorname{Prob}\left(M_{j}=V_{j} \mid \operatorname{abs}\left(s_{j}\right) \geq 11\right) \approx 0.91
$$

and that on the average $\operatorname{abs}\left(s_{j}\right) \geq 11$ for about $44 \%$ of the entries. Note that an attacker knows the vector $s$, which he can compute from the matrix $A$. Thus, an attacker can exploit that for large entries of $s$ the corresponding entry in the secret key $V$ is known with a high probability.

\subsection{The Search Algorithm}

A first attempt for an algorithm is the following. Run the simulated annealing algorithm, Figure 1, $t$ times with the energy function (3). In each run, record the candidate vector $V^{\prime}$ which gave rise to the lowest value of the energy function. Find the entries, say the set $I$, which have the same sign in all these $t$ vectors. Run the simulated annealing algorithm again $t$ times, but instead of choosing a random starting vector, let the entries in $I$ have the values of the first $t$ tests; the remaining entries are chosen at random. Record another $t$ vectors and find another set $I$ and repeat the procedure until a solution is found. This algorithm, hereafter called the PPP-search algorithm, has proved very successful for PPP with larger values of $m$ and $n$. The values of $\alpha$ and $\beta$ play a crucial role for the success of the simulated annealing algorithm. Also, the initial values, that is the values of first $t$ randomly chosen starting vectors, are very important for the further progress of the PPP search algorithm. In the tests reported later in the paper, $\alpha=n$ was chosen.

There are several possible variants of the PPP-search algorithm. The following modifications have been tried with varying success. 
1. When in $t$ runs of SA an entry ends up with the same sign $t$ times, this entry is fixed to this value throughout the entire search.

2. Exploit and incorporate Equation 4 and the facts (5) or (6).

3. Repeat the PPP-search algorithm until a sufficiently high number of entries, say $u$, have the same (correct) sign in $t$ runs of the simulated annealing algorithm. If $u$ is big enough, an exhaustive search for the remaining $n-u$ entries might be possible. Alternatively, one can exhaustively fix a subset of the remaining $n-u$ entries and continue the PPP-search algorithm until a solution is found.

The first variant is faster than the original. Once an entry has the same sign in $t$ consecutive runs of SA, the entry is fixed throughout the remaining search. Clearly, if this entry is correct, this variant will improve the search, but conversely if this entry is incorrect and stays fixed, the search will never find the secret key vector $V$. It is still possible, though, that the search will find a vector $V^{\prime} \neq V$, such that $A V^{\prime}=Y$.

A second variant of the PPP-search algorithm is to choose the entries of the majority vector instead of random values in the starting vector of the first $t$ runs of the simulated annealing algorithm. This has the effect that the set $I$ is larger, however the number of incorrectly assigned entries increases. An alternative is to use the majority vector only for entries where $\operatorname{abs}\left(s_{j}\right)$ has a predetermined high value.

In the following we show how an exhaustive search for a remaining set of entries can be done. First, in the PPP-search algorithm record the frequency of the entries in the candidate vectors $V^{\prime}$ from $\mathrm{SA}$ which gave rise to the lowest value of the energy function. Let $W$ be a vector with $n$ integer entries. After each run of SA set $W=W+V^{\prime}$. After sufficiently many runs of the above algorithm, tests show that $W$ holds the correct sign for a substantial part of the entries of the correct secret key $V$. Assume the search has found about $0<u<n$ correct entries in the candidate solution vector. It turns out that when $u$ is not too small, e.g., if $u>n / 4$, the vector $W$ has the correct sign in 85-90\% of all entries. That is, let $V$ be the chosen solution vector; if $W_{i}<0\left(W_{i}>0\right)$ then with probability $0.85 \ldots 0.90, V_{i}=-1\left(V_{i}=1\right)$. In other words, only $10-15 \%$ of the remaining $n-u$ entries are wrong. Thus if $n-u$ is not too big, it is possible to exhaustively try to determine which of these entries are not in accordance with the sign of $W$. Assume that $u$ entries have been found, and that for $n_{w}$ entries in $W$ it holds that $W_{i} \cdot V_{i}<0$, whereas $W_{i} \cdot V_{i}>0$ for the remaining entries. Then, if $n_{w}$ is known, an exhaustive search at this point takes no longer than

$$
\left(\begin{array}{c}
n-u \\
n_{w}
\end{array}\right)
$$

steps. An attacker cannot know the exact value of $n_{w}$, so the exhaustive search must be repeated for a few values of $n_{w}$ in the neighbourhood of $0.85 \times n$. Alternatively, one can do an exhaustive search of a subset of the remaining $n-u$ entries. The idea is to fix a subset of entries and continue the PPP-search for a 


\begin{tabular}{|cccc|}
\hline $\mathrm{m}$ & $\mathrm{n}$ & Running time & Pointcheval's estimate \\
\hline 101 & 117 & 0.3 & 85 \\
121 & 137 & 0.5 & 130 \\
151 & 167 & 1.5 & 180 \\
\hline
\end{tabular}

Table 1. Running time in seconds for the PP search algorithm (averaged over 50 tests).

number of steps. When the entries in the subset are assigned correct values, the PPP-search will find a solution.

The search can be further improved by incorporating the Equation 4 . When doing the exhaustive search for a few entries, one starts by assigning values to the entries, $j$, for which $\operatorname{abs}\left(s_{j}\right)$ are small. After the assignment of a few entries the remaining entries are either forced by Eq. 4 or lead to contradiction. It is assumed that with this improvement the exhaustive search part takes less than $\left(\begin{array}{c}n-u-1 \\ n_{w}\end{array}\right)$ operations.

As shown in the next section the PPP-search algorithm with $u=n$ works well on instances of PPP where $m \geq n$. In the case where $m<n$, as recommended by Pointcheval, the algorithm is less successful. This may be due to the fact that for a given matrix $A$ and a multiset $S$ there are several solutions to the problem and the search algorithm is not able to converge to one single solution. This is supported by fact that in the cases where $m>n$ the probability is high that there is only a single solution and the algorithms terminate fast and with a high probability of success.

In the cases where $m<n$ the probability of success can be increased by choosing $u<n$ and performing also the exhaustive search part of the algorithm.

\section{Test Results}

The computer used for the tests in this section is a Sun Sparc Ultra-1. When running times are given in seconds or minutes, this is the real time it took the tests to succeed when running on a UNIX system with shared resources. Thus, when implementing on a single dedicated machine, the expected time of the algorithms will be lower.

\subsection{Results for PP}

The PPP-search algorithm can also be used to find solutions for PP by using the energy function (3). For this, PPP-search variant no. 1 choosing $g_{1}=30$ and $g_{2}=0$ in the energy function has proved very successful. Table 1 lists the results of 50 tests on PP with the recommended values of $m$ and $n$. The running time is taken as the total real time divided by 50. All tests succeeded and found a solution. 


\begin{tabular}{|ccccc|}
\hline $\mathrm{m}$ & $\mathrm{n}$ & Tests & Solutions & Running time \\
\hline 73 & 73 & 50 & 19 & $12 \mathrm{~min}$. \\
81 & 81 & 50 & 11 & $22 \mathrm{~min}$. \\
101 & 101 & 50 & 5 & $84 \mathrm{~min}$. \\
101 & 81 & 50 & 32 & $2 \mathrm{~min}$. \\
121 & 81 & 50 & 43 & 1 min. \\
\hline
\end{tabular}

Table 2. Results of the fast search algorithm for PPP. Running time is the average time of all successful tests.

Note that our results listed in Table 1 for PP also improve the bounds determined in 5] for the complexity of average instances of PPP by the same factors. E.g., for $m=101, n=117$, our experiments together with the estimates in [5] give a complexity of $2^{56}$ elementary operations. However, depending on the instance, our experiments with Fast Search as described in the next section show that this complexity may be much lower.

\subsection{PPP - Fast Search}

When implementing attacks on instances on PPP we found that some solutions were found much faster than others. In this section the results of a series of "fast tests" are given. The attacks do not find the solution of all instances of PPP, but when they find a solution, it is found fast. As mentioned earlier, PPP-search variant no. 1 is faster than the original one. When in $t$ runs of the simulated annealing algorithm an entry holds the same sign in the $t$ output vectors, such an entry is fixed is future tests. In these tests, whenever the search algorithm fixed an entry in the candidate solution vector different from the chosen one, the algorithm aborted. These test results are therefore very pessimistic. It might very well be that when the search algorithm was aborted it was converging towards a solution different from the chosen one. Table 2 lists the results of tests on several instantiations of PPP. The running times are pessimistic, taken as the total real time of all 50 tests divided by the number of correct solutions found. In [5] it was reported that no solutions were found for tests on instances of PPP with $m, n>71$, even after running several months. Our results show that in about $40 \%$ of the cases a solution can be found for $m=n=73$ in just 12 minutes running time. The cases $m=101, n=81$ and $m=121, n=81$ are included to illustrate how well the PPP-search algorithm works when $m>n$.

For the target version $m=101, n=117$ the PPP-search algorithm variant no. 1 was implemented with $t=30, \alpha=117$ and $\beta=0.97$ in the simulated annealing part. In 100 tests the algorithm found a solution in one of the cases. Using the exhaustive search extension, the solution can be found much faster than estimated in [5] in 9 of the tests. Table 3 lists the results of 100 tests. In one test the solution was found using about $2^{31}$ simple operations. In one other test 72 entries were found when the search stopped. At this point the vector $W$ held the correct sign in 102 of the 117 entries. Thus, an exhaustive search for the 


\begin{tabular}{|ccccc|}
\hline $\mathrm{m}$ & $\mathrm{n}$ & Tests & Solutions & Complexities (estimates) \\
\hline 101 & 117 & 100 & 9 & $2^{31}, 2^{38}, 2^{43}, 2^{45}, \ldots, 2^{52}$ \\
\hline
\end{tabular}

Table 3. The results of a fast PPP-search for $m=101, n=117$.

\begin{tabular}{|ccccc|}
\hline $\mathrm{m}$ & $\mathrm{n}$ & \multicolumn{3}{c|}{ Tests Solutions Complexity (estimated) } \\
\hline 73 & 73 & 20 & 19 & $2^{26}, \ldots, 2^{33}$ \\
101 & 101 & 20 & 14 & $2^{30}, 2^{33}, 2^{43}, \ldots, 2^{46}$ \\
\hline
\end{tabular}

Table 4. The results of advanced search algorithms for PPP. Complexities are the total number of simple operations.

remaining 47 entries can be done in time about $\left(\begin{array}{l}46 \\ 15\end{array}\right) \approx 2^{38}$ simple operations. The complexities of other cases are done similarly. The stated complexities are of the exhaustive search for the remaining $n-u$ entries which is greater than the first part using simulated annealing. Therefore in a real-life situation, when attacking single instances, it may be very advantageous to run a more complex simulated annealing part.

\subsection{PPP - Advanced Search}

In the previous section a solution was found only for a fraction of all instances. Not surprisingly, increasing the complexity of the tests also increases the probability of success. Table 4 lists the results of a series of tests. For the case $m=n=73$ the PPP-search algorithm was used with $t=40$ using variant no. 2. In the simulated annealing algorithm $\alpha=73$ and $\beta=0.85$ was used. First all entries in the candidate vector for which $\operatorname{abs}\left(s_{j}\right) \geq 11$ were fixed $V_{j}^{\prime}=M_{j}$. As mentioned earlier, for these entries these assignments introduce only a few errors. Subsequently, the PPP-search algorithm was run for a certain number of steps. If no solution was found, one of the entries fixed in the beginning was given the opposite sign and the PPP-search algorithm was restarted. After only a small number of restarts a solution was found in 19 of 20 cases. The tests show that a solution for $m=n=73$ can be found in a few hours with a high probability of success. The total number of simple operations of the attacks varied from $2^{26}$ to $2^{33}$. This attack variant might very well be adapted to the cases $m=n=101$ and $m=101, n=117$.

Variant no. 3 for PPP with $m=n=101$ and where $t=40$ was implemented. First the PPP-search algorithm was run with a complexity of maximum $2^{34}$ simple operations. In 2 of 20 tests a solution was found. For the remaining tests a set of 20 entries which were not found by the search were fixed to the correct value of $V$ and the PPP-search was continued. In 12 of the 18 tests a solution was found in at most 200 runs of the SA algorithm after the assignment of the 20 entries. 200 runs of the SA algorithms in these tests equal about $2^{28}$ steps. In a real-life situation one should repeat the procedure for all possible values of the 20 entries, making the total complexity $2^{34}+s * 2^{20}$ steps for $s \leq 2^{28}$. 
However, the guessing of 20 binary values will succeed after about $2^{19}$ attempts and furthermore, the 20 entries are correlated to the majority vector, a fact we did not incorporate in these tests.

To measure the success of our algorithms an SA step of relatively low complexity was chosen, such that the first part of the test could be implemented in reasonable time. In a real-life setting when attacking a single instance of the PPP one would choose the parameters such that the complexity of both parts of the algorithm would be roughly equal. With a more complex SA step (higher values of $\alpha$ and $\beta$ ) one can expect more correct entries to be identified by the time of the brute-force assignments of additional entries. For instances of PPP with $m=n=101$ run the first part of the above attack with a complexity of, say, $2^{40}$, whereafter most tests would find a solution shortly after an assignment of 20 correct entries, such that the total complexity would remain around $2^{40}$ steps. It is conjectured that a solution can be found for a large part of all PPP instances where $m=n=101$ in time at most $2^{40}$.

It is further conjectured that the same variant of the attack is applicable to instances of PPP with $m=101, n=117$ where the first part of the above attack has complexities of about $2^{45}, \ldots, 2^{50}$, which also would be the total complexity of the attack.

\section{Suggestions for Future Work}

As can be seen from the previous sections there are many possible variants of the PPP-search algorithms. The simulated annealing algorithm is very sensitive to the values of the parameters, and a small change in $\beta$ sometimes produces very different results. As an effect also the behaviour of the PPP-search algorithm changes. In tests on PPP with $m=31, n=47$, different sets of solutions were found with different values of the parameters. In the PPP-search algorithm the value of the parameter $t$ is important. For some instances of PPP, solutions are found fast with a low value of $t$, whereas in other instances a higher value of $t$ seems better. It might be possible also to improve the PPP-search algorithm by using more than one energy function. Either alternate between energy functions from one run of the simulated annealing algorithm to another, or use one energy function in $t$ consecutive runs and another energy function in the next $t$ runs.

It is likely that the limits of our methods as determined in this paper are not optimum and that other variants and/or combinations of the parameters will improve the results.

\section{Conclusion}

In this paper it was demonstrated that the identification schemes based on the permuted perceptron problem are several orders of magnitudes less secure than previously believed. Therefore it is recommended not to use these schemes with the suggested smallest parameters. As a consequence these identification schemes 
compare less favorably to other combinatorial schemes. The iterated search methods developed in this paper can be formulated in general terms and thus might be useful also in other combinatorial problems.

\section{References}

1. E. Aarts and J. Korst. Simulated Annealing and Boltzmann Machines. Wiley, New York, 1989.

2. A. Fiat, A. Shamir. How to prove yourself: practical solutions of identification and signature problems. In A.M. Odlyzko, editor, Advances in Cryptology - CRYPTO'86, LNCS 263, pages 186-194. Springer-Verlag, 1987.

3. S. Goldwasser, S. Micali, C. Rackoff. Knowledge complexity of interactive proof systems. In Proceedings of the 17-th ACM Symposium on the Theory of Computing STOC, ACM, pages 291-304, 1985.

4. M. H. Hassoun. Fundamentals of Artificial Neural Networks. MIT Press, London, England, 1995.

5. D. Pointcheval. A new identification scheme based on the perceptrons problem. In L.C. Guillou and J.-J. Quisquater, editors, Advances in Cryptology - EUROCRYPT'95, LNCS 921, pages 319 - 328. Springer Verlag, 1995.

6. D. Pointcheval. Les réseaux de neurones et leurs applications cryptographiques. Tech. rep. Laboratoire d'Informatique de l'École Normale Supérieure, February 1995. LIENS-95-2.

7. G. Poupard. A Realistic Security Analysis of Identification Schemes based on Combinatorial Problems. European Transactions on Telecommunications, vol. 8, Nr. 5, pages 471-480, 1997.

8. A. Shamir. An efficient identification scheme based on permuted kernels. In G. Brassard, editor, Advances in Cryptology - CRYPTO'89, LNCS 435, pages 606609. Springer-Verlag, 1990.

9. J. Stern. A new identification scheme based on syndrome decoding. In D.R. Stinson, editor, Advances in Cryptology - CRYPTO'93, LNCS 773, pages 13-21. SpringerVerlag, 1994.

10. J. Stern. Designing identification schemes with keys of short size. In Y.G. Desmedt, editor, Advances in Cryptology - CRYPTO'94, LNCS 839, pages 164-173. SpringerVerlag, 1994. 